\title{
Residual Symmetries in the Presence of an EM Background
}

\section{Moises Rojas Leyva*}

Centro Brasileiro de Pesquisas Físicas, Rio de Janeiro, Brazil

E-mail: mrojasecbpf.br

\section{Hector Leny Carrion}

Centro Brasileiro de Pesquisas Físicas, Rio de Janeiro, Brazil

E-mail: hleny@cbpf.br

\section{Francesco Toppan}

Centro Brasileiro de Pesquisas Físicas, Rio de Janeiro, Brazil

E-mail: toppan@cbpf.br

\begin{abstract}
The symmetry algebra of a QFT in the presence of an external EM background (named "residual symmetry") is investigated within a Lie-algebraic, model independent scheme. Some results previously encountered in the literature are here extended. In particular we compute the symmetry algebra for a constant EM background in $\mathrm{D}=3$ dimensions. In $\mathrm{D}=3$ dimensions the residual symmetry algebra, for generic values of the constant EM background, is isomorphic to $u(1) \oplus \mathcal{P}_{c}(2)$, with $\mathcal{P}_{c}(2)$ the centrally extended 2-dimensional Poincaré algebra. Residual symmetry algebras are also computed in the supersymmetric case for a constant EM background. The supersymmetry generators are given by the "square roots" of the deformed translations.
\end{abstract}

Fourth International Winter Conference on Mathematical Methods in Physics 09 - 13 August 2004

Centro Brasileiro de Pesquisas Fisicas (CBPF/MCT), Rio de Janeiro, Brazil

${ }^{*}$ Speaker. 


\section{Introduction}

The issues of QFTs in an external (constant) EM background gained interest recently with the Seiberg and Witten's observation [1] that an ordinary theory in a constant EM background can be reformulated as a non-commutative gauge theory.

The problem of determining the symmetry algebra of a QFT in a constant EM background has been addressed and solved, for the very specific two-dimensional free massive complex boson model minimally coupled to an external gauge field, in [2]. It was proven in that work that its symmetry algebra coincides with the centrally extended Poincaré algebra in $1+1$ dimension, previously investigated in a series of papers [3,4].

In the present work we extend the results of [2]. By using solely Lie-algebraic and modelindependent methods we compute the symmetry algebra of different classes of QFTs coupled to a given external EM background. Throughout the text we call such symmetry algebras "residual symmetries". It is worth stressing that, due to the presence of central extensions, the residual symmetries are not subalgebras of the original symmetry algebra in the absence of the external EM background (such an algebra is given by the direct sum of the Poincaré algebra and a global $U(1)$ charge).

More specifically, we prove that the residual symmetry algebra of a three-dimensional Poincaré invariant QFT in a generic $(|E| \neq|B|)$ constant EM background is given by the 5-dimensional solvable Lie algebra $u(1) \oplus \mathcal{P}_{c}(2)$, where $\mathcal{P}_{c}(2)$ is the two-dimensional centrally extended Poincaré algebra whose signature, Euclidean or Minkowskian, is determined by the relative strength of the constant external electric versus magnetic field (the special case in which the external electric field equals the magnetic field, see [5]).

Furthermore, we extend this result to compute the residual superalgebra for a supersymmetric theory in the presence of a constant EM background. We show that the supersymmetry generators of the residual supersymmetry are the "square roots" of the deformed translations.

The scheme of the paper is the following. In the next section we illustrate the Lie-algebraic method which allows, in a model-independent manner, to determine the residual symmetry generators and the corresponding algebra. In section 3 we present the resulting residual symmetry algebra for a $D=3$ QFT in the presence of a constant EM field. In section 4 the supersymmetric case is treated for the special case of a free theory in $2+1$ dimensions minimally coupled to the external $A_{\mu}$ field describing the constant EM background. Finally, in the Conclusions, we make some comments about our work.

\section{Residual symmetries and their generators}

Let us discuss in detail for the sake of simplicity the case of the residual symmetry for generic Poincaré-invariant field theories in $(2+1)$-dimension, coupled with an external constant EM background.

In the absence of the external electric and magnetic field, the action $\mathcal{S}$ is assumed to be invariant under a 7-parameter symmetry, given by the six generators of the $(2+1)$-Poincaré symmetry which, when acting on scalar fields (the following discussion however is valid no matter which is the spin 
of the fields) are represented by

$$
\begin{aligned}
P_{\mu} & =-i \partial_{\mu}, \\
M_{\mu v} & =i\left(x_{\mu} \partial_{\nu}-x_{v} \partial_{\mu}\right),
\end{aligned}
$$

(the metric is chosen to be +-- ), plus a remaining symmetry generator corresponding to the internal global $U(1)$ charge that will be denoted as $Z$.

It is further assumed that in the action $\mathcal{S}$ the dependence on the classical background field is expressed in terms of the covariant gauge-derivatives

$$
D_{\mu}=\partial_{\mu}-i e A_{\mu}
$$

with $e$ the electric charge.

In the presence of constant external electric and magnetic fields, the $F^{\mu \nu}=\partial^{\mu} A^{\nu}-\partial^{v} A^{\mu}$ fieldstrength is constrained to satisfy

$$
F^{0 i}=E^{i}, \quad F^{i j}=\varepsilon^{i j} B,
$$

where $\mu, v=0,1,2$ and $i, j=1,2$. The fields $E^{i}$ and $B$ are constant. Without loss of generality the $x^{1}, x^{2}$ spatial axis can be rotated so that $E^{1} \equiv E, E^{2}=0$. Throughout the text this convention is respected.

In order to recover (2.2), the gauge field $A_{\mu}$ must depend at most linearly on the coordinates $x^{0} \equiv t, x^{1} \equiv x$ and $x^{2} \equiv y$.

The gauge-transformation

$$
A_{\mu} \mapsto A_{\mu}{ }^{\prime}=A_{\mu}+\frac{1}{e} \partial_{\mu} \alpha\left(x^{v}\right)
$$

allows to conveniently choose for $A_{\mu}$ the gauge-fixing

$$
\begin{aligned}
A_{0} & =0, \\
A_{i} & =E_{i} t-\frac{B}{2} \varepsilon_{i j} x^{j} .
\end{aligned}
$$

The above choice is a good gauge-fixing since it completely fixes the gauge (no gauge-freedom is left). It will be soon evident that the residual symmetry is a truly physical symmetry, independent of the chosen gauge-fixing.

Due to (2.4), the action $\mathcal{S}$ explicitly depends on the $x^{\mu}$ coordinates entering $A_{\mu}$. The simplest way to compute the symmetry property of an action such as $\mathcal{S}$ which explicitly depends on the coordinates consists in performing the following trick. At first $A_{\mu}$ is regarded on the same foot as the other fields entering $\mathcal{S}$ and assumed to transform as standard vector field under the global Poincaré transformations, namely

$$
A_{\mu}^{\prime}\left(x^{\rho^{\prime}}\right)=\Lambda_{\mu}^{v} A_{v}\left(x^{\rho}\right)
$$

for $x^{\mu^{\prime}}=\Lambda^{\mu}{ }_{v} x^{\nu}+a^{\mu}$.

For a generic infinitesimal Poincaré transformation, however, the transformed $A_{\mu}$ gauge-field no longer respects the gauge-fixing condition (2.4). In the active transformation viewpoint only 
fields are entitled to transform, not the space-time coordinates themselves. $A_{\mu}$ plays the role of a fictitious field, inserted to take into account the dependence of the action $S$ on the space-time coordinates caused by the non-trivial background. Therefore, the overall infinitesimal transformation $\delta A_{\mu}$ should be vanishing. This result can be reached if an infinitesimal gauge transformation (2.3) $\delta_{g}\left(A_{\mu}\right)$ can be found in order to compensate for the infinitesimal Poincaré transformation $\delta_{P}\left(A_{\mu}\right)$, i.e. if the following condition is satisfied

$$
\delta\left(A_{\mu}\right)=\delta_{P}\left(A_{\mu}\right)+\delta_{g}\left(A_{\mu}\right)=0 .
$$

Only those Poincaré generators which admit a compensating gauge-transformation satisfying (2.6) provide a symmetry of the $\mathcal{S}$ action (and therefore enter the residual symmetry algebra). This is a plain consequence of the original assumption of the Poincare and manifest gauge invariance for the action $S$ coupled to the gauge-field $A_{\mu}$.

Notice that the original Poincare generators are deformed by the presence of extra-terms associated to the compensating gauge transformation. Let $p$ denote a generator of (2.1) which "survives" as a symmetry in the presence of the external background. The effective generator of the residual symmetry is

$$
\hat{p}=p+(\ldots),
$$

where $(. .$.$) denotes the extra terms arising from the compensating gauge transformation associated$ to $p$. Such $(\ldots)$ extra terms are gauge-fixing dependent. The "residual symmetry generator" $\hat{p}$ can only be expressed in a gauge-dependent manner. However, two gauge-fixing choices are related by a gauge transformation $\mathbf{g}$. The residual symmetry generator in the new gauge-fixing, denoted as $\tilde{p}$, is related to the previous one by an Adjoint transformation

$$
\tilde{p}=\mathbf{g} \hat{p} \mathbf{g}^{-1} \text {. }
$$

Therefore the residual symmetry algebra does not dependent on the choice of the gauge fixing and is a truly physical characterization of the action $\mathcal{S}$, see [5] for details.

\section{The residual symmetry for the $(2+1)$ Poincaré case}

The residual symmetry algebra of the $(2+1)$-Poincare theory involves, besides the global $U(1)$ generator $Z$, the three deformed translations and just one deformed Lorentz generator (the remaining two Lorentz generators are broken).

Within the (2.4) gauge-fixing choice the deformed translations are explicitly given by

$$
\begin{aligned}
P_{0} & =-i \partial_{t}+e E x, \\
P_{1} & =-i \partial_{x}-\frac{e}{2} B y, \\
P_{2} & =-i \partial_{y}+\frac{e}{2} B x .
\end{aligned}
$$

The deformed generator of the residual Lorentz symmetry is explicitly given, in the same gaugefixing and for $E \neq 0$, by

$$
M=i\left(x \partial_{t}+t \partial_{x}\right)-i \frac{B}{E}\left(y \partial_{x}-x \partial_{y}\right)-\frac{e}{2}\left(E t^{2}+E x^{2}+B t y\right) .
$$


The residual symmetry algebra is given by

$$
\begin{aligned}
& {\left[P_{0}, P_{1}\right]=-i E Z} \\
& {\left[P_{0}, P_{2}\right]=0} \\
& {\left[P_{1}, P_{2}\right]=i B Z} \\
& {\left[M, P_{0}\right]=-i P_{1}} \\
& {\left[M, P_{1}\right]=-i P_{0}+i \frac{B}{E} P_{2},} \\
& {\left[M, P_{2}\right]=-i \frac{B}{E} P_{1} .}
\end{aligned}
$$

The $U(1)$ charge $Z$ is no longer decoupled from the other symmetry generators. It appears instead in (3.3) as a central charge.

Please notice that the residual symmetry algebra in $(1+1)$ dimensions (computed in [2] for a specific model) is recovered from the $P_{0}, P_{1}, M, Z$ subalgebra. It corresponds to the centrally extended $2 D$ Poincaré algebra thoroughly studied in [4].

The 5-generator solvable, non-simple Lie algebra of residual symmetries admits a convenient presentation. The generator

$$
\tilde{Z} \equiv B P_{0}-E P_{2}
$$

not only commutes with all the other $*$ generators

$$
[\tilde{Z}, *]=0,
$$

for $|E| \neq|B|$ it is not even present in the r.h.s., so that the residual symmetry algebra is given by a direct sum of $u(1)$ and a 4-generator algebra. The latter algebra is isomorphic to the centrally extended two-dimensional Poincaré algebra. Such an algebra is of Minkowskian or Euclidean type according to whether $|E|>|B|$ or respectively $|E|<|B|$. This point can be intuitively understood due to the predominance of the electric or magnetic effect (in the absence of the electric field the theory is manifestly rotational invariant, so that the Lorentz generator is associated with the Euclidean symmetry). We have explicitly, for $|B|>|E|$, that the algebra

$$
\begin{aligned}
& {\left[\bar{M}, S_{1}\right]=i S_{2},} \\
& {\left[\bar{M}, S_{2}\right]=-i S_{1}}
\end{aligned}
$$

is reproduced by

$$
\begin{aligned}
& \bar{M}=-\frac{E}{\sqrt{B^{2}-E^{2}}} M, \\
& S_{1}=P_{0}-\frac{B}{E} P_{2}, \\
& S_{2}=-\frac{\sqrt{B^{2}-E^{2}}}{E} P_{1},
\end{aligned}
$$

while for $|E|>|B|$ the algebra

$$
\begin{aligned}
& {\left[\tilde{M}, T_{1}\right]=i T_{2},} \\
& {\left[\tilde{M}, T_{2}\right]=i T_{1},}
\end{aligned}
$$


is reproduced by

$$
\begin{aligned}
\tilde{M} & =-\frac{E}{\sqrt{E^{2}-B^{2}}} M, \\
T_{1} & =P_{0}-\frac{B}{E} P_{2}, \\
T_{2} & =\frac{\sqrt{E^{2}-B^{2}}}{E} P_{1} .
\end{aligned}
$$

In both cases the commutator between the translation generators $S_{1}, S_{2}$, and respectively $T_{1}, T_{2}$, develops the central term proportional to $Z$ which can be conveniently normalized.

The residual symmetry algebra of the $(2+1)$ case for generic values of $E$ and $B$ is therefore given by the direct sum

$$
u(1) \oplus \mathcal{P}_{c}(2)
$$

One can easily prove that (3.3) is the residual symmetry algebra even for $E= \pm B$ (see [5]).

\section{The residual supersymmetry}

It is worth investigating the role of the residual (super)symmetry for a supersymmetric theory coupled to an external constant EM background. In principle this can be achieved by extending the method discussed in section $\mathbf{2}$ in application to superfields. In particular the gauge field should be described by a vector superfield, see e.g. [6]. Since however one has to work in components and in the presence of auxiliary fields, the computations are much more cumbersome than in the purely bosonic case. A much more pragmatic viewpoint will be adopted here. Features of the residual supersymmetry can already be grasped by investigating a specific model, given by a free complex spinor $\psi$ and a free complex boson $\phi$, supplemented by a complex auxiliary field $F_{\mu}$ and living, as before, in $(2+1)$ dimension. These fields are minimally coupled to an external EM background $\left(\partial_{\mu} \rightarrow \mathcal{D}_{\mu}=\partial_{\mu}-i e A_{\mu}\right)$.

The lagrangian is given by

$$
\mathcal{L}=\bar{\psi} \Gamma^{\mu} \mathcal{D}_{\mu} \psi+\mathcal{D}_{\mu} \phi^{*} \mathcal{D}^{\mu} \phi-\sqrt{2}\left(\mathcal{D}_{\mu} \phi^{*} F^{\mu}+\mathcal{D}_{\mu} \phi F^{\mu *}\right)+F_{\mu} F^{\mu *} .
$$

In the absence of the external EM background the action is off-shell invariant under the supersymmetry transformations

$$
\begin{aligned}
\delta \phi & =\bar{\varepsilon} \psi \\
\delta \psi & =\bar{\varepsilon} \Gamma^{\mu} \partial_{\mu} \phi^{*} \\
\delta F_{\mu} & =\sqrt{2} \bar{\varepsilon} \partial_{\mu} \psi
\end{aligned}
$$

and their hermitian conjugates.

The above transformations close on-shell, giving the supersymmetry algebra

$$
\left[\delta_{1}, \delta_{2}\right]=\left[\bar{\varepsilon}_{2} \Gamma^{\mu} \varepsilon_{1}-\bar{\varepsilon}_{1} \Gamma^{\mu} \varepsilon_{2}\right] \partial_{\mu}
$$


In the presence of a constant EM background, both (4.2) and (4.3) hold, respectively as off-shell invariance and on-shell superalgebra, by replacing $\partial_{\mu}$ with the covariant derivatives $\mathcal{D}_{\mu}$, in terms of the external background field $A_{\mu}$.

It is worth pointing out that the "deformed translations" $P_{\mu}$ in (3.1) $\left(i P_{\mu}=\partial_{\mu}-i e K_{\mu}\right.$, with $\left.K_{0}=-E x, K_{1}=\frac{B}{2} y, K_{2}=-\frac{B}{2} x\right)$ do not coincide with the covariant derivatives. They can be regarded as covariant derivatives for the reversed fields $E \rightarrow-E, B \rightarrow-B$. However, under a space-inversion $(x \rightarrow-x, y \rightarrow-y)$ supplemented by a convenient gauge transformation with respect to the (2.4) gauge-fixing (see formula (2.7), with the gauge transformation $\alpha$ explicitly given by $\alpha=\frac{e E}{2} t x$, we can identify the "deformed translations" with the covariant derivatives. Since the supersymmetry transformations in different gauge-fixing are related by Adjoint transformations as (2.7), (4.3) holds for Adjoint-related superalgebra generators regardless of the chosen gauge-fixing.

The main result can be expressed as the statement that the residual supersymmetry generators in the presence of a constant EM background can be regarded as the "square roots" of the corresponding (residual) deformed translations.

\section{Conclusions}

In this work we have extended the results of [2] in two directions. We have shown at first the model-independent, Lie-algebraic arising of the result of [2] (originally computed for the free massive complex boson case in $1+1$ dimension, externally coupled to a constant EM background). We named "residual symmetry" this Lie-algebraic characterization of the invariances of a system in the presence of a non-vanishing external EM background.

For a constant EM background in the generic $|E| \neq|B|$ case the residual symmetry of a $D=3$ theory corresponds to the algebra $u(1) \oplus \mathcal{P}_{c}(2)$, where $\mathcal{P}_{c}(2)$ is the centrally extended $2 D$ Poincaré algebra, widely investigated, both mathematically and in physical applications, in [3] (see also [4]).

We also proved that, in the supersymmetric case, the residual supersymmetry algebra in the presence of a constant EM background closes on the deformed Poincaré translations.

\section{References}

[1] N. Seiberg and E. Witten, String theory and noncommutative geometry, JHEP 09 (1999) 032 [hep-th/9908142].

[2] E. Karat, An Example of Poincare Symmetry with a Central Charge, Phys. Lett. B 445 (1999) 337 [hep-th/9810242].

[3] D. Cangemi and R. Jackiw, Geometric Gravitational Forces on Particles Moving in a Line, Phys. Lett. B 299 (1993) 24 [hep-th/ 9210036 ]; ibid. Quantal Analysis of String Inspired Lineal Gravity wich Matter Fields, B 337 (1994) 271 [hep-th/ 9405119 ].

[4] D. Cangemi and R. Jackiw, Poincare Gauge Theory for Gravitational Forces in (1+1)-Dimensions, Ann. Phys. 225 (1993) 229 [hep-th/9302026].

[5] H. L. Carrion, M. Rojas and F. Toppan, Residual Symmetries in the Presence of an EM Background, Mod. Phys. Lett. A 18 (2003) 629 [hep-th/ 0205248].

[6] J. Wess and J. Bagger, Supersymmetry and Supergravity. Princeton Univ. Press, Princeton, 1983. 